\title{
3) Organic Mercury Poisoning
}

\author{
Haruhiko ToKUOMı \\ The First Department of Internal Medicine, Kumamoto University \\ Medical School
}

It has been proved that the Minamata disease is the poisoning by alkyl compound (AM), and recently phenyl mercury compounds (PM) which are used as agricultural drugs have been brought on public discussion.

This report deals with clinical and experimental studies on these poisonings.

\section{Clinical studies}

In Minamata disease, i. e., AM poisoning, concentric constriction of visual fields, hypacusia, disturbance of speech, gait and sensation and tremor etc. were recognized in 80 to $100 \%$ of the cases at the onset of the disease, and these symptoms with less frequency and degree still remain until present, about 10 years after the onset of the disease. It is interesting that a case resembling spastic paraplegia was seen in this study.

The author has never experienced a case of PM poisoning except a patient who took $50 \mathrm{cc}$. of $6.6 \%$ solution of PM compound ( $\mathrm{Hg}: 1.25 \mathrm{~g}$.) with suicide intent. In this case clinical symptoms were observed along with the laboratory examination and biopsy of the kidney, the findings of which were all within normal limits despite a large amount of mercury being estimated in urine. It was recognized that the values for mercury of the hair of farmers was higher than those of control inhabitants.

\section{Experimental studies}

All cats with daily administration of 2 to $4 \mathrm{mg}$. of six kinds of methyl or ethyl mercuric compounds showed the signs of ataxia and convulsion within 20 to 30 days. On the other hand, daily administration of each $0.5,1.0,2.0$, or $3.0 \mathrm{mg}$. of phenyl mercuric acetate (PMA) into rats during three months could not produce any signs of nervous involvement. In these animals the amount of mercury excreted in feces exceeded that in urine. The excretion both in urine and feces increased in the last stage of the administration, so it was thought that the accumulation of mercury in the body might occur in the initial stage. On organ distribution concentrations of mercury in animals with AM poisoning in various organs were in the order to liver, hair, kidney, and brain and those in animals with PM poisoning were kidney, hair, liver and brain. 
Histological study revealed that AM mainly involved the nervous system, being demonstrated such as a fall of granule cells in cerebellum and degeneration and loss of neurons in the cortex with the slight changes in the other organs, while PM produced the minimal injuries in each organ. 